\title{
An Integral Collocation Approach Based on Legendre Polynomials for Solving Riccati, Logistic and Delay Differential Equations
}

\author{
M. M. Khader1,2, A. M. S. Mahdy3 , M. M. Shehata3 \\ ${ }^{1}$ Department of Mathematics and Statistics, College of Science, Al-Imam Mohammad Ibn Saud Islamic \\ University (IMSIU), Riyadh, KSA \\ ${ }^{2}$ Department of Mathematics, Faculty of Science, Benha University, Benha, Egypt \\ ${ }^{3}$ Department of Mathematics, Faculty of Science, Zagazig University, Zagazig, Egypt \\ Email: mohamedmbd@yahoo.com, amr mahdy85@yahoo.com, dr.maha 32@hotmail.com
}

Received 28 May 2014; revised 4 July 2014; accepted 16 July 2014

Copyright (C) 2014 by authors and Scientific Research Publishing Inc.

This work is licensed under the Creative Commons Attribution International License (CC BY).

http://creativecommons.org/licenses/by/4.0/

(c) (i) Open Access

\begin{abstract}
In this paper, we propose and analyze some schemes of the integral collocation formulation based on Legendre polynomials. We implement these formulae to solve numerically Riccati, Logistic and delay differential equations with variable coefficients. The properties of the Legendre polynomials are used to reduce the proposed problems to the solution of non-linear system of algebraic equations using Newton iteration method. We give numerical results to satisfy the accuracy and the applicability of the proposed schemes.
\end{abstract}

\section{Keywords}

Integral Collocation Formulation, Spectral Method, Riccati, Logistic and Delay Differential Equations

\section{Introduction}

It is well known that the ordinary differential equations (ODEs) have been the focus of many studies due to their frequent appearance in various applications, such as in fluid mechanics, viscoelasticity, biology, physics and engineering applications, for more details, for example [1]-[5]. Consequently, considerable attention has been given to the efficient numerical solutions of ODEs of physical interest, because it is difficult to find exact solutions. Different numerical methods have been proposed in the literature for solving ODEs [6]-[13].

The Riccati differential equation (RDE) is named after the Italian Nobleman Count Jacopo Francesco Riccati 
(1676-1754). The book of Reid [14] contains the fundamental theories of Riccati equation, with applications to random processes, optimal control, and diffusion problems. Besides important engineering science applications that today are considered classical, such as stochastic realization theory, optimal control, robust stabilization, and network synthesis, the newer applications include such areas as financial mathematics [15]. The solution of this equation can be reached using classical numerical methods such as, the forward Euler method and RungeKutta method. Bahnasawi et al. [16] presented the usage of Adomian decomposition method to solve the nonlinear RDE in an analytic form. Tan and Abbasbandy [17] employed the analytic technique called homotopy analysis method to solve the quadratic RDE.

The Logistic model can be obtained by applying the derivative operator on the Logistic equation. The model is initially published by Pierre Verhulst in 1838 [18]. The continuous Logistic model is described by first order ODE. The discrete Logistic model is simple iterative equation that reveals the chaotic property in certain regions [19]. There are many variations of the population modeling [20]. The Verhulst model is the classic example to illustrate the periodic doubling and chaotic behavior in dynamical system [19]. The model describs the population growth may be limited by certain factors like population density [20].

\section{Applications of Logistic Equation}

A typical application of the Logistic equation is a common model of population growth. Let $u(t)$ represents the population size and $t$ represents the time where the constant $\rho>0$ defines the growth rate.

Another application of Logistic curve is in medicine, where the Logistic differential equation is used to model the growth of tumors. This application can be considered an extension of the above mentioned use in the framework of ecology.

The solution of Logistic equation is explained the constant population growth rate which not includes the limitation on food supply or spread of diseases [21]. The solution curve of the model is increase exponentially from the multiplication factor up to saturation limit which is maximum carrying capacity [21], $\frac{\mathrm{d} N}{\mathrm{~d} t}=\rho N\left(1-\frac{N}{K}\right)$ where $N$ is the population with respect to time, $\rho$ is the rate of maximum population growth and $K$ is the carrying capacity. The solution of continuous Logistic equation is in the form of constant growth rate as in formula $N(t)=N_{0} \mathrm{e}^{\rho t}$ where $N_{0}$ is the initial population [22].

A delay differential equation (DDE) is a differential equation in which the derivative of the function at any time depends on the solution at previous time. Introduction of delay in the model enriches its dynamics and allows a precise description of the real life phenomena. DDEs are proved useful in control systems [23], lasers, traffic models [24], metal cutting, epidemiology, neuroscience, population dynamics [25], chemical kinetics [26], etc. In DDE, one has to provide history of the system over the delay interval $[-\tau, 0]$ as the initial condition. Due to this reason delay systems are infinite dimensional in nature. Because of in infinite dimensionality the DDEs are difficult to analyze analytically and hence the numerical solutions play an important role.

In [27], Mai-Duy, et al. derived an integral collocation approach based on Chebyshev polynomials and used it for solving numerically the bi-harmonic equations. In [28], Bhrawy and Alofi introduced a new shifted Chebyshev operational matrix of fractional integration of arbitrary order and applied together with spectral tau method for solving linear fractional differential equations. Khader et al. [29] introduced a new approximate formula of the fractional derivative using Legendre series expansion and used it to solve numerically the fractional diffusion equation.

In this article, we extend the previous work and derive some schemes of the integral collocation formulation based on Legendre polynomials. We implement these formulae to solve numerically Riccati, Logistic and delay differential equations.

Our paper is organized as follows: In Section 2, we derive some integration collocation formulations using Legendre series expansion. In Section 3, we give the integral collocation approach for solving Riccati, Logistic and delay differential equations. In Section 4, the paper ends with a brief conclusion and some remarks.

\section{Integration Collocation Formulations}

The well known Legendre polynomials are defined on the interval $[-1,1]$ and can be determined with the aid of the following recurrence formula [30] 


$$
L_{k+1}(z)=\frac{2 k+1}{k+1} z L_{k}(z)-\frac{k}{k+1} L_{k-1}(z), k=1,2, \cdots,
$$

where $L_{0}(z)=1$ and $L_{1}(z)=z$. In order to use these polynomials on the interval [0,1], we define the so called shifted Legendre polynomials by introducing the change of variable $z=2 x-1$. Let the shifted Legendre polynomials $L_{k}(2 x-1)$ be denoted by $L_{k}^{*}(x)$. Then $L_{k}^{*}(x)$ can be obtained as follows

$$
L_{k+1}^{*}(x)=\frac{(2 k+1)(2 x-1)}{(k+1)} L_{k}^{*}(x)-\frac{k}{k+1} L_{k-1}^{*}(x),
$$

where $L_{0}^{*}(x)=1$ and $L_{1}^{*}(x)=2 x-1$. The analytic form of the shifted Legendre polynomials $L_{k}^{*}(x)$ of degree $k$ is given by

$$
L_{k}^{*}(x)=\sum_{i=0}^{k}(-1)^{k+i} \frac{(k+i) !}{(k-i) !(i !)^{2}} x^{i}, k=2,3, \cdots .
$$

Note that $L_{k}^{*}(0)=(-1)^{k}$ and $L_{k}^{*}(1)=1$. The orthogonality condition is

$$
\int_{0}^{1} L_{i}^{*}(x) L_{j}^{*}(x) \mathrm{d} x= \begin{cases}\frac{1}{2 i+1}, & \text { for } i=j, \\ 0, & \text { for } i \neq j .\end{cases}
$$

In this work, to consider the differential equation of $n$-th order, we build the integration collocation method using the truncated Legendre series of degree $m$ to represent the $n$-th derivative of the unknown function $u(x)$ in the following manner

$$
\frac{\mathrm{d}^{n} u(x)}{\mathrm{d} x^{n}} \cong \sum_{k=0}^{m} a_{k} L_{k}^{*}(x)=\sum_{k=0}^{m} a_{k} I_{k}^{(n)}(x) .
$$

Using the integration we can obtain the lower-order derivatives and the function itself as follows

$$
\begin{gathered}
\frac{\mathrm{d}^{n-1} u(x)}{\mathrm{d} x^{n-1}} \cong \sum_{k=0}^{m} a_{k} I_{k}^{(n-1)}(x)+c_{1}, \\
\frac{\mathrm{d}^{n-2} u(x)}{\mathrm{d} x^{n-2}} \cong \sum_{k=0}^{m} a_{k} I_{k}^{(n-2)}(x)+c_{1} x+c_{2}, \cdots \\
\frac{\mathrm{d} u(x)}{\mathrm{d} x}=\sum_{k=0}^{m} a_{k} I_{k}^{(1)}(x)+c_{1} \frac{x^{n-2}}{(n-2) !}+c_{2} \frac{x^{n-3}}{(n-3) !}+\cdots+c_{n-2} x+c_{n-1}, \\
u(x)=\sum_{k=0}^{m} a_{k} I_{k}^{(0)}(x)+c_{1} \frac{x^{n-1}}{(n-1) !}+c_{2} \frac{x^{n-2}}{(n-2) !}+\cdots+c_{n-1} x+c_{n},
\end{gathered}
$$

from (1) and (2) we have

$$
\begin{aligned}
& I_{k}^{(n)}(x)=\sum_{i=0}^{k} \frac{(-1)^{k+i}(k+i) !}{(k-i) !(i !)^{2}} x^{i}, \\
& I_{k}^{(n-1)}(x)=\int I_{k}^{(n)}(x) \mathrm{d} x=\sum_{i=0}^{k} \frac{(-1)^{k+i}(k+i) !}{(k-i) !(i !)^{2}(i+1)} x^{i+1}, \\
& I_{k}^{(n-2)}(x)=\int I_{k}^{(n-1)}(x) \mathrm{d} x=\sum_{i=0}^{k} \frac{(-1)^{k+i}(k+i) !}{(k-i) !(i !)^{2}(i+1)(i+2)} x^{i+2}, \\
& \ldots \\
& I_{k}^{(0)}(x)=\int I_{k}^{(1)}(x) \mathrm{d} x=\sum_{i=0}^{k} \frac{(-1)^{k+i}(k+i) ! x^{i+n}}{(k-i) !(i !)^{2}(i+1) \cdots(i+n-1)(i+n)} .
\end{aligned}
$$


We now collocate Equatuions (2)-(6) at $(m+1)$ points $x_{p}, \quad p=0,1, \cdots, m$ as

$$
\begin{aligned}
& \frac{\mathrm{d}^{n} u\left(x_{p}\right)}{\mathrm{d} x^{n}}=\Omega^{(n)} \hat{S}, \quad \frac{\mathrm{d}^{n-1} u\left(x_{p}\right)}{\mathrm{d} x^{n-1}}=\Omega^{(n-1)} \hat{S}, \cdots, \\
& \frac{\mathrm{d} u\left(x_{p}\right)}{\mathrm{d} x}=\Omega^{(1)} \hat{S}, \quad u\left(x_{p}\right)=\Omega^{(0)} \hat{S},
\end{aligned}
$$

where $\hat{S}=\left[a_{0}, a_{1}, \cdots, a_{m}, c_{1}, c_{2}, \cdots, c_{n}\right]^{\mathrm{T}}$, and $\Omega^{(n)}, \Omega^{(n-1)}, \cdots, \Omega^{(0)}$ are integrated matrices.

\section{Integral Collocation Approach for Solving Riccati, Logistic and Delay Differential Equations}

In this section, we introduce the integral collocation approach using Legendre expansion for solving the Riccati, Logistic and delay differential equations.

\subsection{Model 1: Riccati Differential Equation}

$$
\frac{\mathrm{d} u(x)}{\mathrm{d} x}+u^{2}(x)-1=0, \quad x>0,
$$

we also assume an initial condition

$$
u(0)=u^{0} .
$$

The exact solution to this problem at $u^{0}=0$ is

$$
u(x)=\frac{\mathrm{e}^{2 x}-1}{\mathrm{e}^{2 x}+1} .
$$

The procedure of the implementation is given by the following steps:

1) Approximate the function $u(x)$ using Formula (6) and its relevant derivatives with $m=5$, as follows

$$
\begin{aligned}
& \frac{\mathrm{d} u(x)}{\mathrm{d} x} \cong \sum_{k=0}^{5} a_{k} I_{k}^{(1)}(x), \\
& u(x) \cong \sum_{k=0}^{5} a_{k} I_{k}^{(0)}(x)+c_{1},
\end{aligned}
$$

where $I_{k}^{(0)}(x)$ is defined in (7) as

$$
\begin{aligned}
& I_{k}^{(0)}(x)=\sum_{i=0}^{k}(-1)^{k+i} \frac{(k+i) !}{(k-i) !(i !)^{2}(i+1)} x^{i+1}, \\
& I_{k}^{(1)}(x)=\sum_{i=0}^{k}(-1)^{k+i} \frac{(k+i) !}{(k-i) !(i !)^{2}} x^{i} .
\end{aligned}
$$

Then the Riccati differential Equation (9) is transformed to the following approximated form

$$
\sum_{k=0}^{5} a_{k} L_{k}^{*}(x)+\left(\sum_{k=0}^{5} a_{k} I_{k}^{(0)}(x)+c_{1}\right)^{2}=1
$$

We now collocate Equation (12) at $(m+1=6)$ points $x_{p}, \quad p=0,1,2,3,4,5$ as

$$
\sum_{k=0}^{5} a_{k} L_{k}^{*}\left(x_{p}\right)+\left(\sum_{k=0}^{5} a_{k} I_{k}^{(0)}\left(x_{p}\right)+c_{1}\right)^{2}=1 .
$$

For suitable collocation points we use the roots of shifted Legendre polynomial $L_{6}^{*}(x)$.

2) Also, by substituting from the initial condition (10) in (11) we can obtain $(n=1)$ an equation which gives the value of the constant $c_{1}$ as follows 


$$
c_{1}=u^{0}=0 .
$$

Equations (13) and (14) represent a system of non-linear algebraic equations which contains seven equations for the unknowns $a_{k}, k=0,1,2,3,4,5$ and $c_{1}$.

3) Solve the resulting system using the Newton iteration method to obtain the unknowns $a_{k}, k=0,1,2,3,4,5$ as follows

$$
\begin{aligned}
& a_{0}=0.76160, a_{1}=-0.31790, a_{2}=-0.0506, \\
& a_{3}=0.02896, a_{4}=-0.00111, a_{5}=-0.00112 \text {. }
\end{aligned}
$$

Therefore, from Formula (12) we can obtain the approximate solution in the form

$$
\begin{aligned}
& u(x) \cong \sum_{k=0}^{5} a_{k} I_{k}^{(0)}(x)+c_{1} \\
& s=0.990 x+0.002 x^{2}-0.346 x^{3}+0.027 x^{4}+0.125 x^{5}-0.047 x^{6} .
\end{aligned}
$$

The numerical results of the proposed problem (9) are given in Figure 1 with different values of $m(m=5,7)$ in the interval $[0,1]$ at $u^{0}=0$. From this figure, since the obtained numerical solutions are in excellent agreement with the exact solution, so, we can conclude that the proposed technique is well for solving such class of ODEs.

\subsection{Model 2: Logistic Differential Equation}

$$
\frac{\mathrm{d} u(x)}{\mathrm{d} x}=\rho u(x)(1-u(x)), \quad x>0, \rho>0 .
$$

We also assume an initial condition

$$
u(0)=u_{0}, \quad u_{0}>0 .
$$

The exact solution to this problem is given by

$$
u(x)=\frac{u_{0}}{\left(1-u_{0}\right) \mathrm{e}^{-\rho x}+u_{0}} .
$$

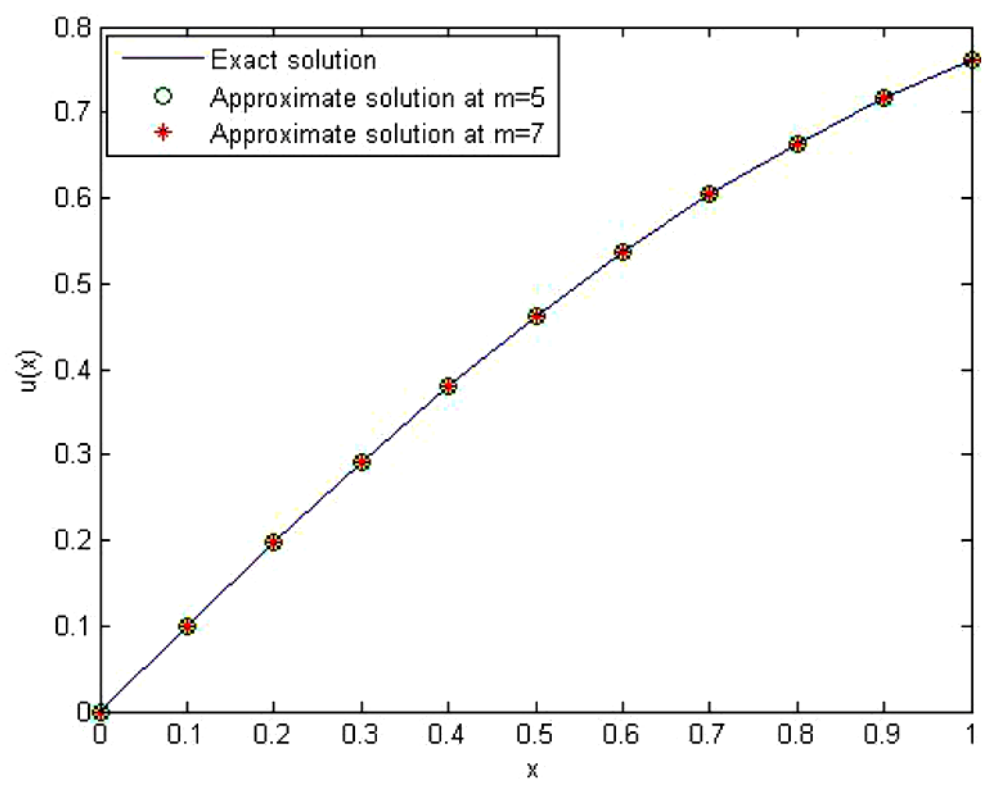

Figure 1. A comparison between the exact solution and the approximate solution at $m=5$ and $m=7$. 
The existence and the uniqueness of the proposed problem (15) are introduced in details in [31].

The procedure of the implementation is given by the following steps:

1) Approximate the function $u(x)$ and its relevant derivatives with $m=5$, using Formula (11). Then the Logistic differential Equation (15) is transformed to the following approximated form

$$
\begin{aligned}
& \sum_{k=0}^{5} a_{k} L_{k}^{*}(x)-\rho\left(\sum_{k=0}^{5} a_{k} I_{k}^{(0)}(x)+c_{1}\right) \\
& \left(1-\left(\sum_{k=0}^{5} a_{k} I_{k}^{(0)}(x)+c_{1}\right)\right)=0 .
\end{aligned}
$$

We now collocate Equation (17) at $(m+1=6)$ points $x_{p}, \quad p=0,1,2,3,4,5$ as

$$
\sum_{k=0}^{5} a_{k} L_{k}^{*}\left(x_{p}\right)-\rho\left(\sum_{k=0}^{5} a_{k} I_{k}^{(0)}\left(x_{p}\right)+c_{1}\right)\left(1-\left(\sum_{k=0}^{5} a_{k} I_{k}^{(0)}\left(x_{p}\right)+c_{1}\right)\right)=0 .
$$

For suitable collocation points we use roots of shifted Legendre polynomial $L_{6}^{*}(x)$ which are

$$
\begin{aligned}
& x_{0}=0.96623, \quad x_{1}=0.03377, \\
& x_{2}=0.38069, x_{3}=0.61930, \\
& x_{4}=0.16931, \quad x_{5}=0.83060 .
\end{aligned}
$$

2) Also, by substituting from the initial condition (16) in (11) with $u_{0}=0.85$ we can obtain $(n=1)$ an equation which gives the value of the constant $c_{1}=0.85$.

Equation (18) represents a system of non-linear algebraic equations which contains six equations for the unknowns $a_{k}, k=0,1,2,3,4,5$.

3) Solve the resulting system using the Newton iteration method to obtain the unknowns $a_{k}, k=0,1,2,3,4,5$ as follows

$$
\begin{aligned}
& a_{0}=0.0533, \quad a_{1}=-0.0101, \\
& a_{2}=0.0004, a_{3}=0.00002, \\
& a_{4}=-1.642 \times 10^{-6}, a_{5}=5.398 \times 10^{-8} .
\end{aligned}
$$

Therefore, from Formula (11) we can obtain the approximate solution in the form

$$
\begin{aligned}
u(x) & =\sum_{k=0}^{5} a_{k} I_{k}^{(0)}(x)+c_{1} \\
& =1+x+0.5 x^{2}+0.1667 x^{3}+0.0417 x^{4}+0.0083 x^{5}+0.0014 x^{6}+0.0002 x^{7}+0.00004 x^{8} .
\end{aligned}
$$

The numerical results of the proposed problem (15) are given in Figure 2 with different values of $m(m=3,5)$ in the interval $[0,1]$. From this figure, since the obtained numerical solutions are in excellent agreement with the exact solution, so, we can conclude that the proposed technique is well for solving such class of ODEs.

\subsection{Model 3: Delay Differential Equation}

Consider the linear delay differential equation of third-order

$$
\frac{\mathrm{d}^{3} u(x)}{\mathrm{d} x^{3}}=-u(x)-u(x-0.3)+\mathrm{e}^{-x+0.3}, 0 \leq x \leq 1,
$$

with the initial conditions

$$
u(0)=1, \frac{\mathrm{d} u(0)}{\mathrm{d} x}=-1, \frac{\mathrm{d}^{2} u(0)}{\mathrm{d} x^{2}}=1, u(x)=\mathrm{e}^{-x} .
$$

The exact solution of this model is $u(x)=\mathrm{e}^{-x}$.

The procedure of the implementation is given by the following steps:

1) Approximate the function $u(x)$ using Formula (6) and its relevant derivatives with $m=5$, as follows 


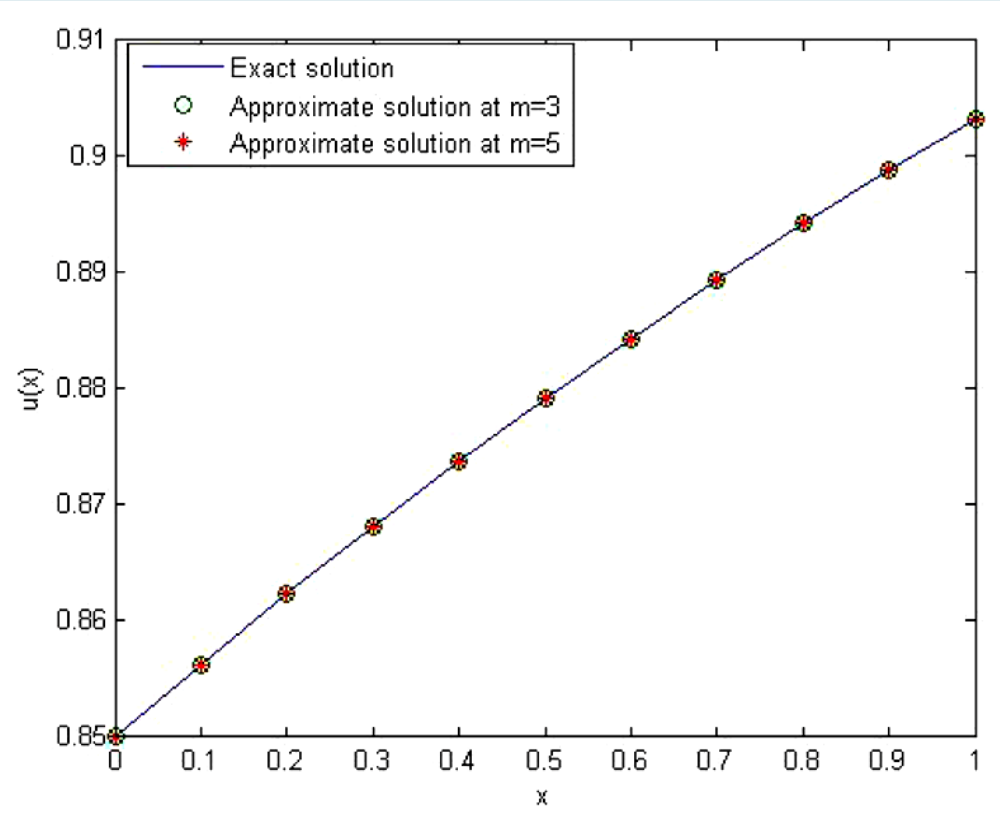

Figure 2. A comparison between the exact solution and the approximate solution at $m=3$ and $m=5$.

$$
\begin{aligned}
& \frac{\mathrm{d}^{3} u(x)}{\mathrm{d} x^{3}} \cong \sum_{k=0}^{5} a_{k} L_{k}^{*}(x)=\sum_{k=0}^{3} a_{k} I_{k}^{(3)}(x), \\
& \frac{\mathrm{d}^{2} u(x)}{\mathrm{d} x^{2}} \cong \sum_{k=0}^{5} a_{k} I_{k}^{(2)}(x)+c_{1}, \\
& \frac{\mathrm{d} u(x)}{\mathrm{d} x} \cong \sum_{k=0}^{5} a_{k} I_{k}^{(1)}(x)+c_{1} x+c_{2}, \\
& u(x) \cong \sum_{k=0}^{5} a_{k} I_{k}^{(0)}(x)+c_{1} \frac{x^{2}}{2 !}+c_{2} \frac{x}{1 !}+c_{3},
\end{aligned}
$$

where $I_{k}^{(0)}(x), \quad I_{k}^{(1)}(x)$ and $I_{k}^{(x)}(x)$ are defined in (7) as follows

$$
\begin{aligned}
& I_{k}^{(0)}(x)=\sum_{i=0}^{k} \frac{(-1)^{k+i}(k+i) !}{(k-i) !(i !)^{2}(i+1)(i+2)(i+3)} x^{i+3}, \\
& I_{k}^{(1)}(x)=\sum_{i=0}^{k} \frac{(-1)^{k+i}(k+i) !}{(k-i) !(i !)^{2}(i+1)(i+2)} x^{i+2}, \\
& I_{k}^{(2)}(x)=\sum_{i=0}^{k} \frac{(-1)^{k+i}(k+i) !}{(k-i) !(i !)^{2}(i+1)} x^{i+1} .
\end{aligned}
$$

Then the delay differential Equation (21) is transformed to the following approximated form

$$
\begin{aligned}
& \sum_{k=0}^{5} a_{k} L_{k}^{*}(x)+\left(\sum_{k=0}^{5} a_{k} I_{k}^{(0)}(x)+c_{1} \frac{x^{2}}{2 !}+c_{2} \frac{x}{1 !}+c_{3}\right) \\
& +\left(\sum_{k=0}^{5} a_{k} I_{k}^{(0)}(x-0.3)+c_{1} \frac{(x-0.3)^{2}}{2 !}+c_{2} \frac{(x-0.3)}{1 !}+c_{3}\right)=\mathrm{e}^{-x+0.3} .
\end{aligned}
$$

We now collocate Equation (24) at $(m+1=6)$ points $x_{p}, \quad p=0,1,2,3,4,5$ as 


$$
\begin{aligned}
& \sum_{k=0}^{5} a_{k} L_{k}^{*}\left(x_{p}\right)+\left(\sum_{k=0}^{5} a_{k} I_{k}^{(0)}\left(x_{p}\right)+c_{1} \frac{x_{p}^{2}}{2 !}+c_{2} \frac{x_{p}}{1 !}+c_{3}\right)+\left(\sum_{k=0}^{5} a_{k} I_{k}^{(0)}\left(x_{p}\right)\left(x_{p}-0.3\right)+c_{1} \frac{\left(x_{p}-0.3\right)^{2}}{2 !}\right. \\
& \left.+c_{2} \frac{\left(x_{p}-0.3\right)}{1 !}+c_{3}\right)=\mathrm{e}^{-x_{p}+0.3} .
\end{aligned}
$$

For suitable collocation points we use the roots of shifted Legendre polynomial $L_{6}^{*}(x)$.

2) Also, by substituting from the initial conditions (22) in (23) we can obtain $(n=3)$ equations which give the values of the constants

$$
c_{1}=1, \quad c_{2}=-1, \quad c_{3}=1
$$

Equations (25) and (26) represent a system of linear algebraic equations which contains nine equations for the unknowns $a_{k}, k=0,1,2,3,4,5$ and $c_{1}, c_{2}, c_{3}$.

3) Solve the resulting system using the conjugate gradient method to obtain the unknowns $a_{k}, k=0,1,2,3,4,5$ as follows

$$
\begin{aligned}
& a_{0}=-0.6321, \quad a_{1}=0.3109, \quad a_{2}=-0.0515, \\
& a_{3}=0.0051, \quad a_{4}=0.0004, \quad a_{5}=0.00002 .
\end{aligned}
$$

Therefore, from Formula (23) we can obtain the approximate solution in the form

$$
\begin{aligned}
u(x) & \cong \sum_{k=0}^{5} a_{k} I_{k}^{(0)}(x)+c_{1} \frac{x^{2}}{2 !}+c_{2} \frac{x}{1 !}+c_{3} \\
& =1-x+0.5 x^{2}-0.1667 x^{3}+0.0417 x^{4}-0.0084 x^{5}+0.0014 x^{6}-0.0002 x^{7}+0.00002 x^{8} .
\end{aligned}
$$

The numerical results of the proposed problem (21) are given in Figure 3 with different values of $m(m=5,7)$ in the interval [0,1]. From this figure, since the obtained numerical solutions are in excellent agreement with the exact solution, so, we can conclude that the proposed technique is well for solving such class of ODEs.

\section{Conclusion and Remarks}

In this article, an integral collocation approach based on Legendre polynomials is introduced for solving

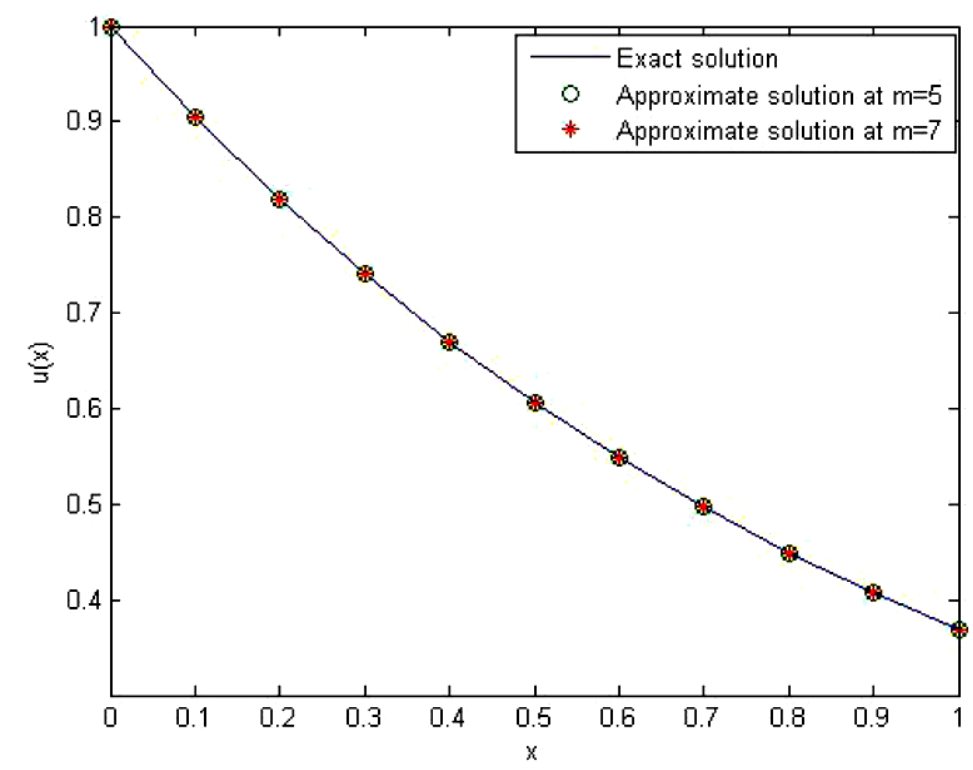

Figure 3. A comparison between the exact solution and the approximate solution at $m=5$ and $m=7$. 
numerically the Riccati, Logistic and delay differential equations. The properties of the Legendre polynomials are used to reduce the proposed problems to system of algebraic equations which are solved by a suitable numerical method. From the obtained numerical results, we can conclude that this method gives results with an excellent agreement with the exact solution. All numerical results are obtained using Matlab program 8.

\section{References}

[1] Khader, M.M. (2011) On the Numerical Solutions for the Fractional Diffusion Equation. Communications in Nonlinear Science and Numerical Simulation, 16, 2535-2542. http://dx.doi.org/10.1016/j.cnsns.2010.09.007

[2] Khader, M.M. (2013) Numerical Treatment for Solving the Perturbed Fractional PDEs Using Hybrid Techniques. Journal of Computational Physics, 250, 565-573. http://dx.doi.org/10.1016/j.jcp.2013.05.032

[3] Sweilam, N.H., Khader, M.M. and Mahdy, A.M.S. (2013) Numerical Study for the Fractional Differential Equations Generated by Optimization Problem Using Chebyshev Collocation Method and FDM. Applied Mathematics and Information Science, 7, 2013-2020.

[4] Khader, M.M. and Hendy, A.S. (2013) A Numerical Technique for Solving Fractional Variational Problems. Mathematical Methods in Applied Sciences, 36, 1281-1289. http://dx.doi.org/10.1002/mma.2681

[5] Sweilam, N.H., Khader, M.M. and Nagy, A.M. (2011) Numerical Solution of Two-Sided Space-Fractional Wave Equation Using Finite Difference Method. Journal of Computional and Applied Mathematics, 235, 2832-2841. http://dx.doi.org/10.1016/j.cam.2010.12.002

[6] Sweilam, N.H., Khader, M.M. and Adel, M. (2012) On the Stability Analysis of Weighted Average Finite Difference Methods for Fractional Wave Equation. Fractional Differential Calculus, 2, 17-75. http://dx.doi.org/10.7153/fdc-02-02

[7] Sweilam, N.H. and Khader, M.M. (2010) A Chebyshev Pseudo-Spectral Method for Solving Fractional Order IntegroDifferential Equations. ANZIAM, 51, 464-475. http://dx.doi.org/10.1017/S1446181110000830

[8] Sweilam, N.H., Khader, M.M. and Al-Bar, R.F. (2008) Homotopy Perturbation Method for Linear and Nonlinear System of Fractional Integro-Differential Equations. International Journal of Computational Mathematics and Numerical Simulation, 1, 73-87.

[9] Sweilam, N.H., Khader, M.M. and Mahdy, A.M.S. (2012) On the Numerical Solution for the Linear Fractional KleinGordon Equation Using Legendre Pseudospectral Method. International Journal of Mathematics and Computer Applications Research, 2, 1-10.

[10] Khader, M.M., EL-Danaf, T.S. and Hendy, A.S. (2013) A Computational Matrix Method for Solving Systems of High Order Fractional Differential Equations. Applied Mathematical Modelling, 37, 4035-4050. http://dx.doi.org/10.1016/j.apm.2012.08.009

[11] Khader, M.M., Mahdy, A.M.S. and Shehata, M.M. (2014) Approximate Analytical Solution to the Time-Fractional Biological Population Model Equation. Jokull, 64, 378-394.

[12] Sweilam, N.H., Khader, M.M. and Mahdy, A.M.S. (2012) Numerical Studies for Solving Fractional-Order Logistic Equation. International Journal of Pure and Applied Mathematics, 78, 1199-1210.

[13] Sweilam, N.H., Khader, M.M. and Mahdy, A.M.S. (2012) Numerical Studies for Fractional-Order Logistic Differential Equation with Two Different Delays. Journal of Applied Mathematics, 2012, Article ID: 764894.

[14] Reid, W.T. (1972) Riccati Differential Equations Mathematics in Science and Engineering. Academic Press, New York.

[15] Lasiecka, I. and Triggiani, R. (1991) Differential and Algebraic Riccati Equations with Application to Boundary/Point Control Problems: Continuous Theory and Approximation Theory. Lecture Notes in Control and Information Sciences, Springer, Berlin.

[16] Bahnasawi, A.A., El-Tawil, M.A. and Abdel-Naby, A. (2004) Solving Riccati Differential Equation Using ADM. Applied Mathematics and Computation, 157, 503-514. http://dx.doi.org/10.1016/j.amc.2003.08.049

[17] Tan, Y. and Abbasbandy, S. (2008) Homotopy Analysis Method for Quadratic Riccati Differential Equation. Communications in Nonlinear Science and Numerical Simulation, 13, 539-546. http://dx.doi.org/10.1016/j.cnsns.2006.06.006

[18] Cushing, J.M. (1998) An Introduction to Structured Population Dynamics, Society for Industrial and Applied Mathematics. http://dx.doi.org/10.1137/1.9781611970005

[19] Alligood, K.T., Sauer, T.D. and Yorke, J.A. (1996) An Introduction to Dynamical Systems. Springer, Berlin.

[20] Ausloos, M. The Logistic Map and the Route to Chaos: From the Beginnings to Modern Applications XVI. 411, 2006.

[21] Pastijn, H. (2006) Chaotic Growth with the Logistic Model of P.-F. Verhulst, Understanding Complex Systems. The Logistic Map and the Route to Chaos. 3-11. 
[22] Suansook, Y. and Paithoonwattanakij, K. (2009) Dynamic of Logistic Model at Fractional Order. IEEE International Symposium on Industrial Electronics.

[23] Fridman, E., Fridman, L. and Shustin, E. (2000) Steady Modes in Relay Control Systems with Time Delay and Periodic Disturbances. Journal of Dynamic Systems, Measurement, and Control, 122, 732-737.

http://dx.doi.org/10.1115/1.1320443

[24] Davis, C.L. (2002) Modification of the Optimal Velocity Traffic Model to Include Delay due to Driver Reaction Time. Physica A, 319, 557-567. http://dx.doi.org/10.1016/S0378-4371(02)01457-7

[25] Kuang, Y. (1993) Delay Differential Equations with Applications in Population Biology. Academic Press, Boston, San Diego, New York.

[26] Epstein, I. and Luo, Y. (1991) Differential Delay Equations in Chemical Kinetics: Nonlinear Models: The Cross-Shaped Phase Diagram and the Originator. Journal of Chemical Physics, 95, 244-254. http://dx.doi.org/10.1063/1.461481

[27] Mai-Duy, N., See, H. and Tran-Cong, T. (2009) A Spectral Collocation Technique Based on Integrated Chebyshev Polynomials for Biharmonic Problems in Irregular Domains. Applied Mathematical Modelling, 33, 284-299. http://dx.doi.org/10.1016/j.apm.2007.11.002

[28] Bhrawy, A.H. and Alofi, A.S. (2013) The Operational Matrix of Fractional Integration for Shifted Chebyshev Polynomials. Applied Mathematics Letters, 26, 26-31. http://dx.doi.org/10.1016/j.aml.2012.01.027

[29] Khader, M.M., Sweilam, N.H. and Mahdy, A.M.S. (2011) An Efficient Numerical Method for Solving the Fractional Diffusion Equation. Journal of Applied Mathematics and Bioinformatics, 1, 1-12.

[30] Bell, W.W. (1968) Special Functions for Scientists and Engineers. Great Britain, Butler and Tanner Ltd, Frome and London.

[31] El-Sayed, A.M.A., El-Mesiry, A.E.M. and El-Saka, H.A.A. (2007) On the Fractional-Order Logistic Equation. Applied Mathematics Letters, 20, 817-823. http://dx.doi.org/10.1016/j.aml.2006.08.013 
Scientific Research Publishing (SCIRP) is one of the largest Open Access journal publishers. It is currently publishing more than 200 open access, online, peer-reviewed journals covering a wide range of academic disciplines. SCIRP serves the worldwide academic communities and contributes to the progress and application of science with its publication.

Other selected journals from SCIRP are listed as below. Submit your manuscript to us via either submit@scirp.org or Online Submission Portal.
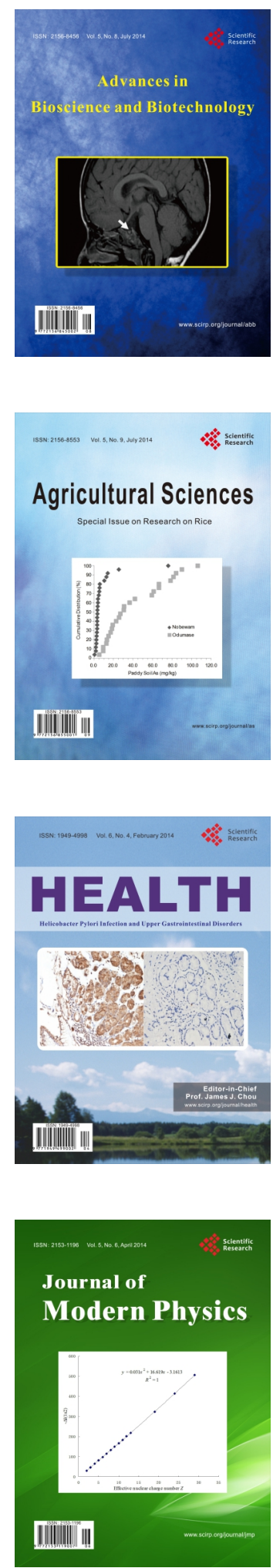
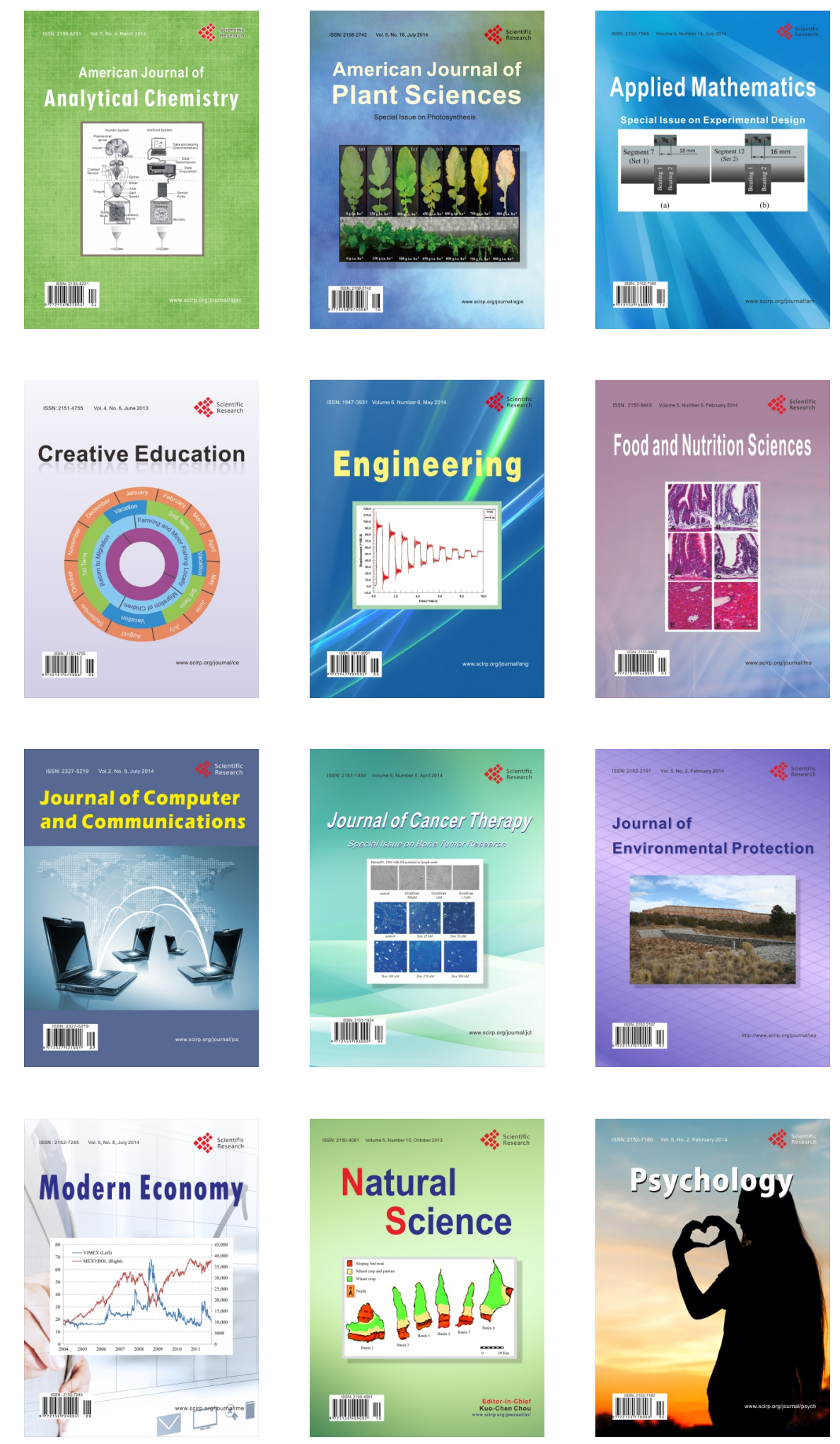